\title{
THE CONNECTEDNESS OF THE COLLECTION OF ARC CLUSTER SETS
}

\author{
BY \\ PETER LAPPAN $\left(^{1}\right)$
}

\begin{abstract}
Let $f$ be a continuous complex-valued function defined on the unit disk and let $p$ be a boundary point of the disk. A very natural topology on the collection of all arc cluster sets of $f$ at the point $p$ has been investigated by Belna and Lappan [1] who proved that this collection is a compact set under certain suitable conditions. It is proved here that this collection is an arcwise connected set under the topology in question, but is not in general locally arcwise connected or even locally connected. It is also shown by example that it is generally not possible to map the real line onto the collection of arc cluster sets at $p$ in a continuous manner.
\end{abstract}

1. Let $D$ denote the open unit disk, let $\Gamma$ denote the unit circle, and for $p \in \Gamma$, let $\mathfrak{I}(p)$ denote the collection of all Jordan arcs in $D \cup\{p\}$ with one endpoint in $D$ and the other at $p$. If $Q$ is a subset of $\bar{D}$ and if $f$ is a complex-valued function defined on $D$, we define the cluster set of $f$ at $p$ relative to $Q$ by

$$
C_{Q}(f, p)=\bigcap_{r>0} \mathrm{Cl}(f(Q \cap N(r, p))),
$$

where $N(r, p)=\{z \in D:|z-p|<r\}$ and the closure is taken relative to the Riemann sphere $W$. Thus each arc $t \in \mathfrak{I}(p)$ determines a nonempty closed set $C_{t}(f, p)$. Let $\mathfrak{C}_{f}(p)=\left\{C_{t}(f, p): t \in \mathfrak{T}(p)\right\}$ be called the collection of all arc cluster sets of $f$ at $p$. Thus $\mathbb{C}_{f}(p)$ is a set for which each element $C_{t}(f, p)$ is itself a closed subset of $W$. If $f$ is a continuous function it is well known that $C_{t}(f, p)$ is a connected subset of $W$ for each $t \in \mathfrak{T}(p)$.

Let $z$ and $z^{1}$ be two points of the Riemann sphere $W$ and let $d\left(z, z^{1}\right)$ denote the chordal distance between $z$ and $z^{1}$. If $A$ is a subset of $W$, let

$$
d(z, A)=\inf \{d(z, a) ; a \in A\}
$$

denote the distance between the point $z$ and the set $A$. If $A$ and $B$ are subsets of $W$, define the $M$-distance between $A$ and $B$ by

$$
M(A, B)=\max \{\sup \{d(a, B): a \in A\}, \sup \{d(b, A): b \in B\}\} .
$$

The $M$-distance defines a Hausdorff topology on the set of all closed subsets of $W$ [2], and hence defines a Hausdorff topology on $\mathfrak{E}_{f}(p)$ for each $p \in \Gamma$ and each complex-valued function $f$.

Received by the editors April 15, 1971 and, in revised form, November 17, 1971.

AMS 1969 subject classifications. Primary 3062; Secondary 5455.

Key words and phrases. Cluster set, arc cluster set, arcwise connectedness.

(1) The author acknowledges partial support from the National Science Foundation under grant GP 11825. 
Lappan [3] has proved that if $f$ is a continuous function in $D$ then, for each $p \in \Gamma, \mathfrak{\complement}_{f}(p)$ is either a singleton or else has continuum many elements. Belna and Lappan [1] have proved that if $f$ is a continuous function in $D$ and if $p \in C$ is not an ambiguous point in the sense of Bagemihl then $\mathfrak{c}_{f}(p)$ is a compact set in the $M$-topology. In particular, this means that if $f$ is continuous then $\mathbb{E}_{f}(p)$ is compact for all but a countable set of points $p \in \Gamma$. In this paper we will consider connectedness properties for the set $\mathfrak{\mho}_{f}(p)$, where $f$ is a continuous function.

2. We begin by defining a concept especially designed for the proofs to follow.

Definition. If $p \in \Gamma$ and if $t \in \mathfrak{T}(p)$, a set $E$ is said to be a tree with trunk $t$ if $E=t \cup \bigcup_{n=1}^{\infty} s_{n}$, where the sets $s_{n}$ satisfy all of the following conditions:

(1) For each $n$, either $s_{n}=\varnothing$ or else $s_{n}$ is a Jordan arc in D having its initial endpoint $p_{n}$ on $t$, but no other point of $t$ lies in $s_{n}$. (The Jordan arc $s_{n}$ need not include its other endpoint, although, of course, it may include this endpoint.)

(2) $s_{n} \cap s_{k}=\varnothing$ for $k \neq n$, and $\bar{s}_{n} \cap\{p\}=\varnothing$ for each $n$.

(3) For each $n$, there exists a real number $r_{n}$ with $0<r_{n}<1$ and $\lim _{n \rightarrow \infty} r_{n}=1$ such that $s_{n} \subset\left\{z \in D:|z|>r_{n}\right\}$.

(4) $\bar{E} \cap \Gamma=\{p\}$.

We remark that the arc $t$ itself is a tree under this definition.

We shall have occasion to refer to what we call a double tree. Let $p \in \Gamma$ and let $t_{0}$ and $t_{1}$ be elements of $\mathfrak{I}(p)$ such that $t_{0} \cap t_{1} \cap D=\varnothing$. It is no loss of generality to assume that both $t_{0}$ and $t_{1}$ have initial points on the circle $\left\{z:|z|=\frac{1}{2}\right\}$, and that all other points of $t_{0} \cup t_{1}$ are contained in $\left\{z:|z|>\frac{1}{2}\right\}$. Let $s_{1}^{\prime}$ be a subarc of the circle $\left\{z:|z|=\frac{1}{2}\right\}$ joining the endpoints of $t_{0}$ and $t_{1}$ such that $t_{0} \cup t_{1} \cup s_{1}^{\prime}$ is the boundary of a bounded region $\Delta$. For each $n>1$, let $s_{n}^{\prime}$ be a subarc of the circle $\{z:|z|=n /(n+1)\}$ which meets both $t_{0}$ and $t_{1}$ and also disconnects the region $\Delta$ in such a way that the component of $\Delta-s_{n}^{\prime}$ having $p$ as a boundary point does not contain $s_{n-1}^{\prime}$. Let $p_{n}$ be the endpoint of $s_{n}^{\prime}$ on $t_{0}$, let $q_{n}$ be the endpoint of $s_{n}^{\prime}$ on $t_{1}$, let $u_{n}$ be the portion of $t_{0}$ between $p_{n}$ and $p_{n+1}$, and let $v_{n}$ be the portion of $t_{1}$ between $q_{n}$ and $q_{n+1}$, where $u_{n}$ includes the point $p_{n}$ but not the point $p_{n+1}$ and $v_{n}$ includes the point $q_{n}$ but not the point $q_{n+1}$. Setting $s_{n}=s_{n}^{\prime} \cup v_{n}$, we have that $E=t_{0} \cup t_{1} \cup \bigcup_{n=1}^{\infty} s_{n}^{\prime}=t_{0} \cup \bigcup_{n=1}^{\infty} s_{n}$ is a tree with trunk $t_{0}$. However, if we set $s_{n}^{\prime \prime}=s_{n}^{\prime} \cup u_{n}$, we have that the same point set $E$ can be expressed as $E=t_{1} \cup \bigcup_{n=1}^{\infty} s_{n}^{\prime \prime}$, so that $E$ may also be considered as a tree with trunk $t_{1}$. We will refer to this point set $E$ as the double tree on $t_{0}$ and $t_{1}$.

Each tree $E$ with trunk $t$ in $\mathfrak{T}(p)$ determines a cluster set $C_{E}(f, p)$ for a given function $f$. To simplify notation, we will use $C E$ in place of $C_{E}(f, p)$ whenever no ambiguity will result.

LEMMA 1. If $p \in \Gamma, t \in \mathfrak{T}(p)$, if $E$ is a tree with trunk $t$, and if $f$ is a continuous function in $D$, then $C E \in \mathfrak{C}_{f}(p)$.

Proof. Let $E=t \cup \bigcup_{n=1}^{\infty} s_{n}$, where the nonempty sets $s_{n}$ are listed in the order in which their initial points $p_{n}$ appear on $t_{0}$. Let $\mu_{n}$ denote the portion of $t_{0}$ between 
$p_{n}$ and $p_{n+1}$, where $\mu_{n}$ includes the point $p_{n}$ but not the point $p_{n+1}$. Then $s_{n} \cup \mu_{n}$ is a Jordan arc (possibly not including either of its endpoints). There exists a Jordan arc $t_{n}$ which has its initial point at $p_{n}$, its terminal point at $p_{n+1}$, and approximates $s_{n} \cup \mu_{n}$ in such a manner that both

$$
\sup \left\{d\left(z, s_{n} \cup \mu_{n}\right): z \in t_{n}\right\}+\sup \left\{d\left(z, t_{n}\right): z \in s_{n} \cup \mu_{n}\right\}<1 / n
$$

and

(6) $\sup \left\{d\left(f(z), f\left(s_{n} \cup \mu_{n}\right)\right): z \in t_{n}\right\}+\sup \left\{d\left(f(z), f\left(t_{n}\right)\right): z \in s_{n} \cup \mu_{n}\right\}<1 / n$.

Furthermore, the arcs $t_{n}$ can be chosen to satisfy (5) and (6) and such that $t_{n} \cap t_{n+1}$ $=\left\{p_{n+1}\right\}$ and $t_{n} \cap t_{j}=\varnothing$ for $j \geqq n+2$. Setting $t=\bigcup_{n=1}^{\infty} t_{n} \cup\{p\}$, we have that $t \in T(p)$ and $C_{t}(f, p)=C E \in \mathbb{E}_{f}(p)$.

Lemma 2. Let $p \in \Gamma, t \in \mathfrak{T}(p)$, and let $E$ and $E^{\prime}$ be two trees with the same trunks $t$ such that $E^{\prime} \subset E$. Let $f$ be a continuous function in $D$ such that $C E^{\prime} \neq C E$. Given $r$, $0<r<1$, there exists a tree $E^{\prime \prime}$ with trunk $t$ satisfying $E^{\prime} \subset E^{\prime \prime} \subset E$ and $M\left(C E^{\prime}, C E^{\prime \prime}\right)$ $=r M\left(C E^{\prime}, C E\right)$.

Proof. We first note that $C E^{\prime} \subset C E$, and if $E^{\prime} \subset E^{\prime \prime} \subset E$, then $C E^{\prime} \subset C E^{\prime \prime} \subset C E$. Let $r$ be given, $0<r<1$, and let $G=\left\{z \in D: d\left(f(z), C E^{\prime}\right)<r M\left(C E^{\prime}, C E\right)\right\}$. If $E^{\prime}=t \cup \bigcup_{n=1}^{\infty} s_{n}^{\prime}$ and $E=t \cup \bigcup_{n=1}^{\infty} s_{n}$, then we may suppose that $s_{n}^{\prime} \subset s_{n}$ for each $n$, since the points of $E$ can be redistributed among the arcs $s_{n}$, if necessary, and the arcs $s_{n}^{\prime}$ can be re-indexed as necessary without changing either tree as a point set. There exists a component $G^{\prime}$ of $G$ and an integer $N$ such that $s_{n}^{\prime} \subset G^{\prime}$ for $n \geqq N$. For $n \geqq N$, let $s_{n}^{\prime \prime}$ be the component of $s_{n} \cap G^{\prime}$ containing $s_{n}^{\prime}$, and for $n<N$ set $s_{n}^{\prime \prime}=s_{n}^{\prime}$. Then, setting $E^{\prime \prime}=t \cup \bigcup_{n=1}^{\infty} s_{n}^{\prime \prime}$, we have $E^{\prime} \subset E^{\prime \prime} \subset E$ and also $M\left(C E^{\prime}, C E^{\prime \prime}\right)$ $=r M\left(C E^{\prime}, C E\right)$, so that $E^{\prime \prime}$ is the desired tree.

Lemma 3. Let $f$ be a continuous function in $D$, let $p \in \Gamma$, let $t \in \mathfrak{T}(p)$, and let $E$ be a tree with trunk $t$. For each $\xi>0$ there exists a finite number of trees $E_{n}, n=0,1$, $2, \ldots, m(\xi)$, each with trunk $t$ such that

$$
t=E_{0} \subset E_{1} \subset E_{2} \subset \cdots \subset E_{m(\xi)}=E
$$

and $M\left(C E_{n}, C E_{n+1}\right)<\xi$ for $0 \leqq n<m(\xi)$.

Proof. Let $\xi>0$ be given and let $j$ be a positive integer such that $j>1 / \xi$. Set $E_{0}=t$. If $M\left(C E_{0}, C E\right) \leqq 1 / j$, then set $E=E_{1}$ and we are finished. If $M\left(C E_{0}, C E\right)$ $>1 / j$, then by a repeated application of Lemma 2 we can construct a sequence $\left\{E_{n}\right\}$ of trees, each with trunk $t$, such that $E_{n} \subset E_{n+1}$ and $M\left(C E_{n}, C E_{n+1}\right)=1 / j$ for each $n$, where we continue the construction as far as possible. Since $C E_{n}$ is a subset of the Riemann sphere $W$, we have that $C E_{n+1}$ contains a point of $W$ a distance $1 / j$ from the set $C E_{n}$. Since $W$ is compact, the construction of the trees $E_{n}$ must terminate after a finite number of steps.

This completes the proof of the lemma. 
LEMMA 4. Let $f$ be a continuous function in $D$, let $p \in \Gamma$, let $t \in \mathfrak{T}(p)$ and let $E$ be a tree with trunk $t$. There exists a countable collection of trees $\left\{E_{n}: n=0,1,2, \ldots\right\}$ each with trunk $t$, with $E_{0}=t$ and $E_{1}=E$ and such that there exists a sequence $\left\{a_{n}\right\}$ of real numbers dense in the interval $[0,1]$ with $a_{0}=0$ and $a_{1}=1$ and such that

(7) if $a_{n}<a_{m}$ then $E_{n}$ is a subset of $E_{m}$;

(8) for each $\xi>0$ there exist integers $j>0, k>1$ such that $M\left(C E_{0}, C E_{j}\right)<\xi$ and $M\left(C E_{k}, C E_{1}\right)<\xi ;$ and

(9) for each real number $x, 0<x<1$, and each $\xi>0$ there exist integers $q$ and $s$ such that $a_{q}<x<a_{s}$ and $M\left(C E_{q}, C E_{s}\right)<\xi$.

Proof. By Lemma 3, we can choose a finite collection $\mathfrak{S}_{1}$ of trees, each with trunk $t$, such that $\mathfrak{S}_{1}=\left\{E_{1, j}: 0 \leqq j \leqq m_{1}\right\}$ with $t=E_{1,0}, E=E_{1, m_{1}}$, and for each $j$, $0 \leqq j<m_{1}$, both $E_{1, j} \subset E_{1, j+1}$ and $M\left(C E_{1, j}, C E_{1, j+1}\right) \leqq \frac{1}{2}$ are satisfied, where the equality holds for all $j$ with the possible exception of $j=m_{1}-1$. By repeated application of Lemma 3, we can find a sequence $\left\{\widetilde{S}_{n}\right\}$ of finite collections of trees each with trunk $t$ satisfying $\mathfrak{S}_{n} \subset \Im_{n+1}, \mathfrak{S}_{n}=\left\{E_{n, j}: 0 \leqq j \leqq m_{n}\right\}, E_{n, 0}=t, E_{n, m_{n}}=E, E_{n, j}$ $\subset E_{n, j+1}$, and $M\left(C E_{n, j}, C E_{n, j+1}\right) \leqq 1 /(n+1)$ for each $n$ and each $j, 0 \leqq j<m_{n}$, where the equality holds for all $n$ and $j$ with the possible exceptions of those $n$ and $j$ for which $E_{n, j+1} \in \mathfrak{S}_{n-1}$.

We now construct the sequence $\left\{a_{n}\right\}$. Let $a_{0}=0, a_{1}=1$, and choose $a_{2}, a_{3}, \ldots, a_{m_{1}}$ to be the points $a_{n+1}=n / m_{1}$. For $2 \leqq j \leqq m_{1}$, assign $a_{j}$ to the tree $E_{1, j-1}$. Proceeding inductively, we assume that to each tree in $\Im_{n}$ we have assigned a unique number $a_{j}$ with the property that each interval in $[0,1]$ of length $1 / n$ contains at least one of the numbers $a_{0}, a_{1}, \ldots, a_{m_{n}}$, and that the order of size of the numbers $a_{j}$ corresponds to the order under set inclusion of the corresponding trees of $\mathfrak{S}_{n}$. We can then assign to each of the $\left(m_{n+1}-m_{n}\right)$ trees in $\mathfrak{S}_{n+1}-\mathfrak{S}_{n}$ a number $a_{j}$, where the number $a_{j}$ is assigned in such a way that the order of size of the numbers $a_{j}, 0 \leqq j \leqq m_{n+1}$, corresponds to the order under set inclusion of the corresponding trees in $\mathbb{S}_{n+1}$, and such that if $k$ new numbers are assigned to an interval $I$ determined by adjacent numbers corresponding to trees in $\Im_{n}$, then the new numbers will divide the interval $I$ into $(k+1)$ intervals of equal length. By this construction, the set $\left\{a_{n}: n=0,1\right.$, $2, \ldots\}$ is a dense subset of the interval $[0,1]$. We may now list the trees in $\bigcup \mathfrak{S}_{n}$ as $E_{0}, E_{1}, E_{2}, \ldots$ in such a way that (7), (8), and (9) are satisfied.

3. We are now in a position to state and prove some results concerning the connectedness of $\mathfrak{c}_{f}(p)$.

THEOREM 1. Let $f$ be a continuous function in $D$, let $p \in \Gamma$, let $t \in \mathfrak{T}(p)$, and let $E$ be a tree with trunk $t$. There exists a continuous function $\pi_{E}$ from $[0,1]$ into $\mathbb{C}_{f}(p)$ such that $\pi_{E}(0)=C_{t}(f, p)$ and $\pi_{E}(1)=C E$.

Proof. Let $\left\{a_{n}\right\}$ and $\left\{E_{n}\right\}$ be as described in Lemma 4. Define $\pi_{E}(x)=\bigcap_{a_{n} \geqq x} C E_{n}$ 
for each $x \in[0,1]$, and let $E(x)=\bigcap_{a_{n} \geqq x} E_{n}$. For $0<x<1$ and $\xi>0$, by (7) and (9) there exist numbers $a_{q}$ and $a_{s}$ such that $a_{q}<x<a_{s}, E_{q} \subset E(x) \subset E_{s}, C E_{q} \subset C E(x)$ $\subset C E_{s}$, and $M\left(C E_{q}, C E_{s}\right)<\xi$. It follows that $C E(x)=\pi_{E}(x)$ so that $\pi_{E}(x) \in \mathfrak{C}_{f}(p)$ by Lemma 1 and $\pi_{E}$ is continuous on the open interval $(0,1)$. By using (8) in place of (9), we obtain in a similar manner that $\pi_{E}$ is continuous at $x=0$ and at $x=1$. Thus $\pi_{E}$ is the desired function.

THEOREM 2. If $f$ is a continuous function in $D$ and if $p \in \Gamma$, then $\mathbb{\mathfrak { F }}_{f}(p)$ is arcwise connected in the M-topology.

Proof. We will show that for each pair of $\operatorname{arcs} t_{1}, t_{2} \in \mathfrak{I}(p)$ there is a continuous function $\pi$ from $[0,1]$ into $\mathcal{E}_{f}(p)$ such that $\pi(0)=C_{t_{1}}(f, p)$ and $\pi(1)=C_{t_{2}}(f, p)$.

If $C_{t_{1}}(f, p)=C_{t_{2}}(f, p)$, then we can define $\pi(x)=C_{t_{1}}(f, p)$ for $0 \leqq x \leqq 1$ and we are finished.

If $C_{t_{1}}(f, p) \neq C_{t_{2}}(f, p)$ and if $t_{1} \cap t_{2}=\{p\}$, then we may assume that $t_{1}$ and $t_{2}$ both originate on the circle $\left\{z:|z|=\frac{1}{2}\right\}$. Let $E$ be the double tree on $t_{1}$ and $t_{2}$ and consider $E$ first as a tree with trunk $t_{1}$. By Theorem 1 there exists a continuous function $\pi_{E}$ from $[0,1]$ into $\mho_{f}(p)$ such that $\pi_{E}(0)=C_{t_{1}}(f, p)$ and $\pi_{E}(1)=C E$. Now let $E^{\prime}=E$, where $E^{\prime}$ is considered as a tree with trunk $t_{2}$. Again, by Theorem 1 , there exists a continuous function $\pi_{E^{\prime}}$ from $[0,1]$ into $\mathfrak{夭}_{f}(p)$ such that $\pi_{E^{\prime}}(0)=C_{t_{2}}(f, p)$ and $\pi_{E^{\prime}}(1)=C E^{\prime}$. The function $\pi(x)$, defined such that $\pi(x)=\pi_{E}(2 x)$ for $0 \leqq x \leqq \frac{1}{2}$ and $\pi(x)=\pi_{E^{\prime}}(2-2 x)$ for $\frac{1}{2} \leqq x \leqq 1$, is a continuous function from $[0,1]$ into $\mathbb{C}_{f}(p)$ with $\pi(0)=C_{t_{1}}(f, p)$ and $\pi(1)=C_{t_{2}}(f, p)$, so that $\pi$ is the desired function in this case.

Finally, if $t_{1} \cap t_{2} \cap D \neq \varnothing$ then there exists an arc $t_{3} \in \mathfrak{I}(p)$ such that $t_{1} \cap t_{3}$ $=\{p\}=t_{2} \cap t_{3}$. By the argument just completed there exist continuous functions $\pi_{1}$ and $\pi_{2}$ from $[0,1]$ into $\widetilde{C}_{f}(p)$ such that $\pi_{1}(0)=C_{t_{1}}(f, p), \pi_{1}(1)=C_{t_{3}}(f, p), \pi_{2}(0)$ $=C_{t_{3}}(f, p)$, and $\pi_{2}(1)=C_{t_{2}}(f, p)$. We obtain the desired function $\pi$ by taking $\pi(x)=\pi_{1}(2 x)$ for $0 \leqq x \leqq \frac{1}{2}$, and $\pi(x)=\pi_{2}(2 x-1)$ for $\frac{1}{2} \leqq x \leqq 1$. This completes the proof of the theorem.

REMARK. The proof of Theorem 2 involves a reasonably smooth shift of trees with a fixed trunk with one "discontinuity" by considering the same point set as different trees with different trunks. The elements of $\mathfrak{T}(p)$ corresponding to these two trees are not at all close together in any intuitive sense. It would seem reasonable that an arc in $\mathfrak{E}_{f}(p)$, i.e. the continuous function $\pi$ in Theorem 2 , would describe a smooth shift in elements of $\mathfrak{T}(p)$, without a "discontinuity" of the sort mentioned. A proof of Theorem 2 involving such a smooth shift, without any "discontinuity" of the type mentioned, would be highly desirable.

Theorem 2 shows that $\mathfrak{S}_{f}(p)$ is an arcwise connected set, and hence a connected set whenever $f$ is continuous. However, it is not true that $\mathfrak{\Im}_{f}(p)$ need be locally connected, much less locally arcwise connected, where $f$ is continuous.

THEOREM 3. There exists a function $f$ continuous in $D$ for which $\mathfrak{\mho}_{f}(1)$ is not locally connected. 
Proof. Let $A_{n}$ be the chord of $D$ given by

$$
A_{n}=\{z \in D: \arg (z-1)=\pi / 2+1 / n\},
$$

let $z_{n}=(1 / n) e^{i / n}$ and let $w_{n}=e^{i / n}, n=1,2,3, \ldots$ Define $f$ on the set $\{z: \arg (z-1) \geqq \pi\}$ by $f(z)=0$ there. For all odd $n$, define $f(z)=z_{n}$ for $z \in A_{n}$, while for all even $n$ define $f(z)=w_{n}$ for $z \in A_{n}$. For these $z$ satisfying $\pi / 2+1 /(n+1)<\arg (z-1)<\pi / 2+1 / n$, define $f(z)=t a_{n}+(1-t) a_{n+1}$ for $\arg (z-1)=\pi / 2+1 /(n+1)+t / n(n+1), 0<t<1$, $n=1,2, \ldots$, where $a_{n}$ and $a_{n+1}$ are the values assumed by $f(z)$ on $A_{n}$ and $A_{n+1}$, respectively. Finally, for $z$ such that $\pi / 2+1<\arg (z-1)<\pi$, define

$$
f(z)=\left(2 e^{i} /(\pi-2)\right)(\pi-\arg (z-1)) .
$$

The function $f$ is now defined as continuous in $D$.

The value 0 is an asymptotic value of $f$ at 1 so $\{0\} \in \mathfrak{C}_{f}(1)$. If $\xi$ is given such that $0<\xi<\frac{1}{2}$, then there exist points $z_{n_{1}}$ and $z_{n_{2}}, n_{1}$ and $n_{2}$ both odd, such that $0<d\left(0, z_{n_{1}}\right)<\xi, 0<d\left(0, z_{n_{2}}\right)<\xi$. But the points $z_{n_{1}}$ and $z_{n_{2}}$ are located in different components of $\{z:|z|<\xi\} \cap C_{D}(f, 1)$. It follows that $\mathfrak{C}_{f}(1)$ is not locally connected.

4. In light of Theorem 2, it would be interesting to be able to list elements of $\mathfrak{c}_{,}(p)$ in a systematic (i.e., continuous) manner. The following result shows that this is not possible.

THEOREM 4. There exists a continuous function $f$ in $D$ for which there is no continuous function from the real line onto $\mathfrak{S}_{f}(1)$.

Proof. Let $P$ denote the Cantor ternary set on $[0,1]$, let $\left\{I_{n}\right\}$ be the open intervals removed from $[0,1]$ to obtain $P$, let $y_{n}$ be the midpoint of $I_{n}$. For each $\theta,-\pi / 2<\theta$ $<\pi / 2$, let $L(\theta)$ be the chord

$$
L(\theta)=\{z \in D: \arg (z-1)=\pi+\theta\} .
$$

For each $n=1,2,3, \ldots$, let

$$
\begin{aligned}
& A_{n}=\left\{z \in D:|z-1| \leqq 1 / n, \arg (z-1)-\pi \in I_{n}\right\}, \\
& B_{n}=\left\{z \in D:|z-1| \geqq 1 /(n-1), \arg (z-1)-\pi \in I_{n}\right\}, \text { and } \\
& C_{n}=\left\{z \in D: 1 / n<|z-1|<1 /(n-1), \arg (z-1)-\pi \in I_{n}\right\} .
\end{aligned}
$$

(Interpret $B_{1}=\varnothing$ and $C_{1}=\left\{z \in D:|z-1|>1\right.$, $\left.\arg (z-1) \in I_{1}\right\}$.) For each $x \in P$, let $S_{x}$ be the line segment from $x$ to the point $\frac{1}{2}+i$ and let $K=\bigcup\left\{S_{x}: x \in P\right\}$.

We begin to define a continuous function $f$ on the unit disk by setting $f(z)=0$ for $z \in L(\theta), \theta<0$, setting $f(z)=1$ for $z \in L(\theta), \theta \geqq 1$, and setting $f(z)=\theta$ for $z \in L(\theta)$, $\theta \in P$. Then $f$ is defined at each $z$ except for those $z$ on some $L(\theta), \theta \in \bigcup I_{n}$. For $\theta \in I_{n}$ and $z \in L(\theta)$, we have $z \in A_{n} \cup B_{n} \cup C_{n}$. If $z \in B_{n}$, set $f(z)=\theta$ and if $z \in L\left(y_{n}\right)$ $\cap A_{n}$, set $f(z)=\frac{1}{2}+i$. Let $x_{n, 1}$ and $x_{n, 2}$ be the right- and left-hand endpoints of $I_{n}$. If $\theta \in\left(x_{n, 1}, y_{n}\right)$ and $z \in L(\theta) \cap A_{n}$, and if $\theta=t x_{n, 1}+(1-t) y_{n}, 0<t<1$, set $f(z)$ $=t x_{n, 1}+(1-t)\left(\frac{1}{2}+i\right)$, while if $\theta \in\left(y_{n}, x_{n, 2}\right)$ and $z \in L(\theta) \cap A_{n}$, and if $\theta=s x_{n, 2}$ $+(1-s) y_{n}, C<s<1$, set $f(z)=s x_{n, 2}+(1-s)\left(\frac{1}{2}+i\right)$. Finally if $\theta \in I_{n}$, and $z \in L(\theta)$ $\cap C_{n}$, let $a=L(\theta) \cap\{z:|z-1|=1 / n\}$ and $b=L(\theta) \cap\{z:|z-1|=1 /(n-1)\}$ and 
letting $z=t a+(1-t) b, 0<t<1$, define $f(z)=t f(a)+(1-t) f(b)$. (For $z \in C_{1}$, take $a=L(\theta)=\{z:|z-1|=1\}$ and set $f(z)=f(a)$.) The function $f$ is now defined on all of $D$ and continuous there, $C_{D}(f, 1)=K$ and for each $x \in P$ we have $\{x\} \in \mathbb{E}_{f}(1)$.

Suppose that $\pi$ is a continuous function for the real line onto $\mathfrak{F}_{f}(1)$. For each $x \in P$, let $t_{x}$ be an element of $\pi^{-1}(x)$. Since the set $P^{1}=\left\{t_{x}: x \in P\right\}$ is uncountable, there exists a point $t^{1}$ which is a limit point of the set $P^{1}$. Let $\left\{t_{n}\right\}$ be a monotone sequence of points in $P^{1}$ converging to $t^{1}$. For each $s$ there must exist a point $t_{n}^{1}$ between $t_{n}$ and $t_{n+1}$ such that $\frac{1}{2}+i \in \pi\left(t_{n}^{1}\right)$ by the continuity of $\pi$. But $\pi\left(t^{1}\right)=$ $\lim _{n \rightarrow \infty} \pi\left(t_{n}\right)$ is a subset of $P$, while $\pi\left(t^{1}\right)=\lim _{n \rightarrow \infty} \pi\left(t_{n}^{1}\right)$ is a continuum containing $\frac{1}{2}+i$. It follows that the continuity of the function $\pi$ is untenable, so the theorem is proved.

Theorem 4 may be interpreted as saying that the topological space $\mathfrak{\mho}_{f}(1)$ is not a Peano continuum.

REMARK. Neither of the examples constructed in the proofs of Theorems 3 and 4 are meromorphic functions. It would be interesting if meromorphic functions satisfying these theorems could be found. The construction of meromorphic functions with the desired properties appears to the author to be very difficult.

5. In what has preceded, we have dealt only with the local situation at a single point $p$. We now make a brief consideration of the global situation.

Let $f$ be a function mapping $D$ conformally onto $D-S$, where $S$ is the spiral $\left\{z=r e^{i \theta}: r=\theta /(\theta+\pi), \theta \geqq \pi\right\}$. There exists a point $p_{0} \in \Gamma$ such that for each $t \in T\left(p_{0}\right)$ we have $C_{t}\left(f, p_{0}\right)=\Gamma$, while for each point $p \in \Gamma-\left\{p_{0}\right\}$ the cluster set $C_{D}(f, p)$ is a singleton set. It follows that $\bigcup\left\{\aleph_{f}(p): p \in \Gamma\right\}$ is not a connected set, so that the most obvious attempt at an analogue to Theorem 2 in a global setting is not valid.

However, if we broaden the family of cluster sets under consideration we can find a valid global version of Theorem 2 . Let $\mathfrak{T}$ be the family of all Jordan arcs $z(t), 0 \leqq t<1$, in $D$ for which $|z(t)| \rightarrow 1$ as $t \rightarrow 1$. Then $\mathfrak{T}$ includes arcs other than those with an endpoint on $\Gamma$. If $f$ is a continuous function in $D$ and $t \in \mathfrak{T}$, let

$$
C_{t}(f)=\bigcap_{0<r<1} \mathrm{Cl}(f(t) \cap\{z: r<|z|<1\})
$$

and let $\mathfrak{C}^{*}(f)=\left\{C_{t}(f): t \in \mathfrak{T}\right\}$. Then Theorem 2 is valid with $\mathfrak{T}$ and $\mathfrak{C}^{*}(f)$ used in place of $\mathfrak{I}(p)$ and $\mathfrak{E}_{f}(p)$, respectively. To prove this we need only modify the definition of a tree by allowing the trunk to be in $\mathfrak{I}$ rather than $\mathfrak{T}(p)$ and by eliminating condition (4) from the definition. It is then easy to modify all of the proofs of the lemmas and Theorems 1 and 2 to obtain the result. It should be noted that Theorems 3 and 4 remain valid with $\mathfrak{T}$ and $\mathfrak{C}^{*}(f)$ in place of $\mathfrak{T}(p)$ and $\mathfrak{C}_{f}(p)$, respectively, because it is easy to modify each of the functions constructed so that $\mathfrak{E}_{f}(1)=\mathfrak{C}^{*}(f)$ for each function. 


\section{REFERENCES}

1. C. L. Belna and P. Lappan, The compactness of the set of arc cluster sets, Michigan Math. J. 16 (1969), 211-214. MR 9, 7111.

2. C. Kuratowski, Topologie. I. Espaces métrisables, espaces complets, 2ième éd., Monografie Mat., Tom 20, PWN, Warsaw, 1948. MR 10, 389.

3. P. Lappan, Continua which are curvilinear cluster sets, Nagoya Math. J. 34 (1969), 25-34. MR 39 \#1660.

Department of Mathematics, Michigan State University, East Lansing, Michigan 48823 